\title{
Human Biomechanical Model Based Optimal Design of Assistive Shoulder Exoskeleton
}

\author{
Marc G. Carmichael, Dikai K. Liu
}

\begin{abstract}
Robotic exoskeletons are being developed to assist humans in tasks such as robotic rehabilitation, assistive living, industrial and other service applications. Exoskeletons for the upper limb are required to encompass the shoulder whilst achieving a range of motion so as to not impede the wearer, avoid collisions with the wearer, and avoid kinematic singularities during operation. However this is particularly challenging due to the large range of motion of the human shoulder. In this paper a biomechanical model based optimisation is applied to the design of a shoulder exoskeleton with the objective of maximising shoulder range of motion. A biomechanical model defines the healthy range of motion of the human shoulder. A genetic algorithm maximises the range of motion of the exoskeleton towards that of the human, whilst taking into account collisions and kinematic singularities. It is shown how the optimisation can increase the exoskeleton range of motion towards that equivalent of the human, or towards a subset of human range of motion relevant to specific applications.
\end{abstract}

\section{Introduction}

Exoskeletons are a type of robot worn by the operator to provide physical assistance. These systems have the potential to significantly benefit numerous industrial and service applications such as nursing [19], agriculture [18], rehabilitation [13], and reduce fatigue or injury [6] in tasks like materials handling as depicted in Figure 1. Exoskeletons are commonly categorised into systems that assist either the upper or the lower limbs. Although applications exist which would benefit from both types, most examples that have reached commercialisation are for the lower limb. It is speculated that a factor contributing to this may be the additional challenges

Marc G. Carmichael and Dikai K. Liu

University of Technology, Sydney. Centre for Autonomous Systems,

e-mail: marc.carmichael@uts.edu.au,dikai.liu@uts.edu.au 
associated with designing an exoskeleton for the human shoulder, which has a large range of motion and is described as the most complicated of the major articulations in the human body [7]. Activities utilising the upper limb require a large amount of dexterity, hence for an exoskeleton to be beneficial a design that does not impede the wearer's natural range of motion (ROM) is required. However designing an exoskeleton that achieves a human equivalent ROM is challenging as it is required to do so whilst encompassing the wearer, maintaining no collision with the wearer, and be satisfactorily far from kinematic singularity throughout its operation.
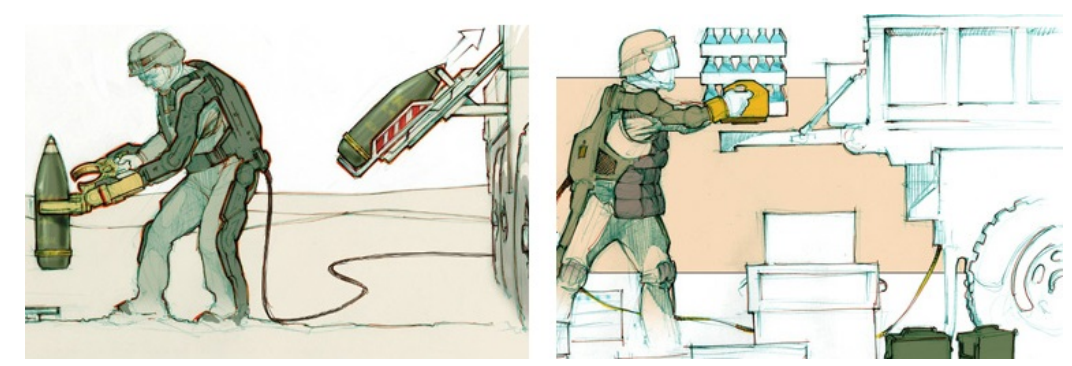

Fig. 1 Depiction of robotic exoskeletons assisting the upper limb in materials handling applications [8]. Copyright 2010 Raytheon Sarcos [17].

Robotic exoskeletons that encompass the shoulder exist in a number of kinematic variations in the literature with ranging levels of complexity and sophistication [9, $16,1,14,3,10]$. When designing the shoulder mechanism a common approach is to focus on the location of the singularity and manually position it outside or at the edge of the desired workspace to maximise the usable range of motion $[9,16,1,2]$. In this work we design a shoulder exoskeleton using an optimisation process that incorporates a biomechanical model of the human arm [12,11]. With the human shoulder ROM defined by the biomechanical model, the design parameters of an exoskeleton are optimised using a genetic algorithm to maximise its ROM towards that of the human, whilst accounting for singularity and collisions. Additionally the optimisation is utilised with a subset of human ROM corresponding to workspace regions relevant to different tasks. This demonstrates how the presented optimisation is a useful tool for adapting new or existing exoskeleton designs to specific industrial or service applications.

\section{Human Shoulder Range of Motion (ROM)}

Maximising exoskeleton ROM towards that equivalent of the human shoulder requires that the ROM of the human shoulder first be defined. The human shoulder ROM is defined as the region of 3D space the humerus can be located. With translation of the shoulder not considered, the ROM is represented simply by all the ori- 
entations the humerus can reach. Many sources in the literature describe shoulder ROM, however these are often isolated to individual planar articulations. A general model of shoulder ROM representing all reachable orientations not limited to distinct articulations is required.

The biomechanical model developed by [12] represents the upper limb, including the shoulder complex, as a serial chain of rigid links connected by ideal joints. This model was developed to determine the reachable workspace of the human wrist, and includes equations describing the limits of each of the joints in the model. This allows the orientation of the humerus to be tested whether or not it is located within these limits, and hence is in a biomechanically reachable orientation. Although this shoulder model consists of five joints representing clavicle, scapula and humerus movement, only three coordinates are used to describe the orientation of the humerus relative to the torso. These are $\phi_{A}$ for abduction/adduction, $\phi_{F}$ for flexion/extension, and $\phi_{R}$ for medial/lateral rotation. Limits for these joints are shown in (1) taken from [12], where $\phi_{A m}, \phi_{A M}, \phi_{F m}, \phi_{F M}, \phi_{R m}$ and $\phi_{R M}$ are constants based on clinical measurements of the subject. These are intended to allow the reachable workspace of patients with upper limb impairments to be calculated. In this work we consider the person wearing the exoskeleton as not having an impairment and hence use the healthy values shown in Table 1 taken from [11] and adapted for the model [12].

$$
\begin{aligned}
\phi_{A} & =\left(\phi_{A m}, \phi_{A M}\right) \\
\phi_{F} & =\left(\phi_{F m}+\frac{1}{3} \phi_{A}, \phi_{F M}-\frac{1}{6} \phi_{A}\right) \\
\phi_{R} & =\left(\phi_{R m}+\frac{7}{9} \phi_{A}-\frac{1}{9} \phi_{F}+\frac{4}{9 \pi} \phi_{A} \phi_{F}, \phi_{R M}+\frac{4}{9} \phi_{A}-\frac{5}{9} \phi_{F}+\frac{10}{9 \pi} \phi_{A} \phi_{F}\right)
\end{aligned}
$$

Table 1 Parameters used in the biomechanical model based on healthy human shoulder [11].

\begin{tabular}{cc|cc|cc}
\hline \multicolumn{2}{c|}{ Abduction/Adduction } & \multicolumn{2}{c|}{ Flexion/Extension } & \multicolumn{2}{c}{ Internal/External rotation } \\
$\phi_{A m}$ & $\phi_{A M}$ & $\phi_{F m}$ & $\phi_{F M}$ & $\phi_{R m}$ & $\phi_{R M}$ \\
\hline$-10^{\circ}$ & $170^{\circ}$ & $-60^{\circ}$ & $170^{\circ}$ & $-90^{\circ}$ & $60^{\circ}$ \\
\hline
\end{tabular}

Representation of the humerus ROM is made by generating a large set of orientations the humerus can reach in the workspace. Later this set is used to evaluate the ROM of the exoskeleton based on the number of orientations the exoskeleton can also satisfactorily reach. One approach to generate this set is to recursively step through the range of each joint in the biomechanical model to produce a set of feasible humerus rotations. A problem with this approach is the resulting set of rotations are not evenly distributed which causes problems when quantifying the ROM that the exoskeleton can reach. Consider the case where the exoskeleton is unable to 
reach a region in the human's ROM. If this region so happens to contain a dense distribution of rotations in the set, then this would skew the calculated exoskeleton ROM. Hence it is essential that an even distribution of rotations be used when quantifying the ROM the exoskeleton can achieve.

To mitigate skewing of the ROM calculation a method that creates a more even distribution is implemented. A set of randomly distributed 3D rotations is created represented by unit quaternions. Since each quaternion contains four parameters with unit length, the set can be treated as points lying on the surface of a 4-dimensional unit sphere. Points on the sphere were constrained to only one hemisphere since points that are diametrically opposite from each other represent identical rotations. Once the random set of quaternions is created, a routine is implemented where the points on the sphere are repelled from each other. This is performed recursively whilst constraining the points to the surface of the sphere until a distribution considered as even is achieved. Figure 2 shows this process before and after using a 3-dimensional sphere equivalent for visualisation purposes.

Fig. 2 Randomly generated points on unit sphere. (a) before the even distribution procedure. (b) after the even distribution procedure. The same procedure is used on a 4D sphere to evenly distribute a set of quaternion rotations.

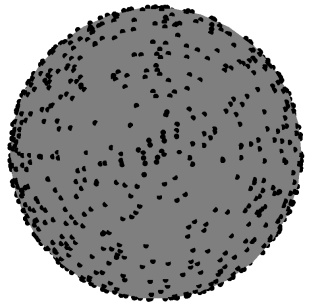

(a) Before routine

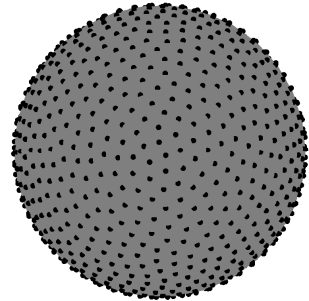

(b) After routine

A set $\psi=\left\{R_{H 1}, R_{H 2}, \cdots, R_{H n}\right\}$ containing 30,000 evenly distributed rotations is made. Each element in this set represents the orientation of the human humerus relative to the torso. A subset of $\psi$ is created by utilising the biomechanical shoulder model to determine if each individual rotation is reachable by the human humerus. Inverse kinematics is performed to solve for the humerus abduction, flexion and rotation angles of the shoulder model, as detailed in the Appendix. If these angles are found to be within range according to (1) then we consider the rotation to be biomechanically feasible. Collision between the humerus and torso is also checked as this is not accounted for in the model [11]. The resulting subset $\psi_{H}$ contains 5,227 humerus rotations which are determined as biomechanically feasible, and have on average a rotation of $7.6^{\circ}$ between neighboring rotations in the set.

\section{Exoskeleton Design}

The exoskeleton design optimised in this work has a configuration similar to some described in the literature $[9,3]$. It consists of three revolute joints interconnected 
by angled links such that their axes of rotation intersect to produce a 3 degree of freedom (DOF) spherical joint. The centre of rotation is coincident with the wearer's glenohumeral joint as shown in Figure 3. When optimising this design it is assumed the exoskeleton is worn by the user, hence link 0 is in a fixed location relative to the torso. Likewise the end effector is attached to the upper arm, hence link 3 is in a fixed location relative to the humerus. The human shoulder complex is not a pure spherical joint as it translates as well as rotates, however the translation is ignored as it is assumed differences in exoskeleton and human arm kinematics can be accommodated by passive compliance between the robot and the wearer [9]. Lastly we assume the radii of the joints and links to the shoulder's centre can be designed after optimisation in such a way that exoskeleton self collision can be avoided, therefore robot collision with itself is not considered during optimisation.
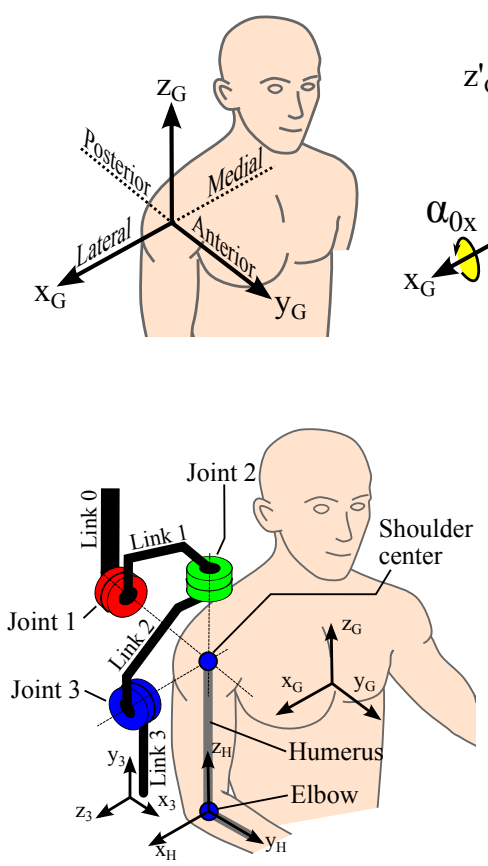

(b)

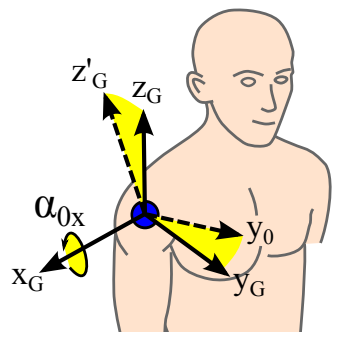

(a)
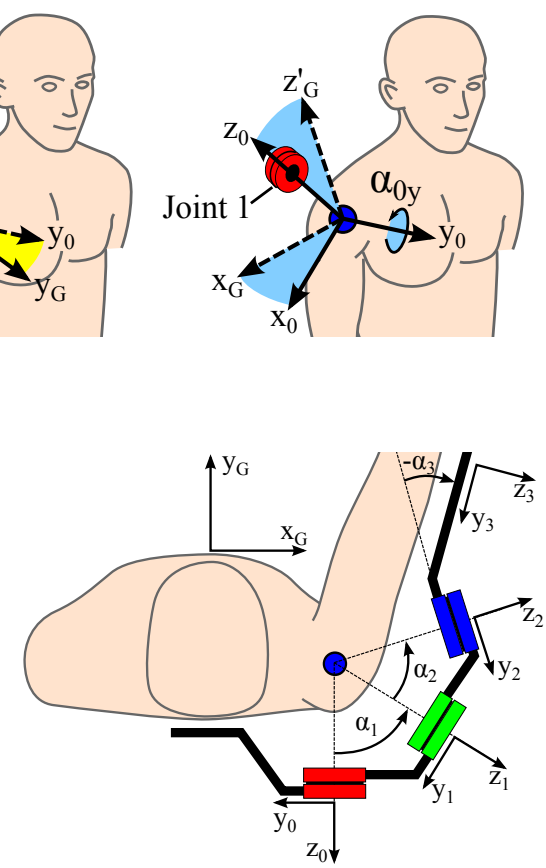

(c)

Fig. 3 (a) Location of exoskeleton joint 1 with respect to the human torso frame $R_{G}$, determined by design parameters $\alpha_{0 x}$ and $\alpha_{0 y}$. (b) Shoulder exoskeleton with links and joints labelled. Coordinate frames for the human torso $\left(R_{G}\right)$, humerus $\left({ }^{G} R_{H}\right)$, and link $3\left({ }^{G} R_{3}\right)$ are shown. (c) Relationship between the global torso frame $R_{G}$ and exoskeleton link frames ${ }^{G} R_{0},{ }^{G} R_{1},{ }^{G} R_{2}$, and ${ }^{G} R_{3}$. The design parameters and joint angles shown in this subfigure are; $\alpha_{0 x}=90^{\circ}, \alpha_{0 y}=0^{\circ}, \theta_{1}=90^{\circ}$, $\theta_{2}=0^{\circ}, \theta_{3}=0^{\circ}$.

Since the mechanism forms a spherical joint the kinematics of the exoskeleton can be expressed solely by rotations. Rotation matrix $R_{G}$ represents the global coordinate frame and is assigned to the human torso with $x$-axis directed laterally, $y$-axis 
anteriorly, and $z$-axis directed superiorly. Orientation of the human humerus is defined by rotation matrix ${ }^{G} R_{H}$, with the superscript denoting that it is represented with respect to the global frame. The humerus frame has $z$-axis pointing from the elbow to the shoulder, $y$-axis pointing from the elbow to the wrist (if the elbow is bent at $90^{\circ}$ right angle), and $x$-axis being their cross product to form a right-handed coordinate system. The relative rotation between $R_{H}$ and the coordinate frame of link 3 remains fixed as shown in Figure $3 b$. Orientation of link 0 is represented by rotation matrix ${ }^{G} R_{0}$ which is parameterised using two successive rotations, $\alpha_{0 x}$ about the $x$-axis followed by $\alpha_{0 y}$ about the $y$-axis (2). The convention of defining each joint axis as coinciding with the $z$-axis of the preceding link's frame is used, hence ${ }^{G} R_{0}$ determines the location of joint 1 .

$$
{ }^{G} R_{0}=\left[\begin{array}{ccc}
\cos \alpha_{0 y} & 0 & \sin \alpha_{0 y} \\
\sin \alpha_{0 x} \sin \alpha_{0 y} & \cos \alpha_{0 x} & -\sin \alpha_{0 x} \cos \alpha_{0 y} \\
-\cos \alpha_{0 x} \sin \alpha_{0 y} & \sin \alpha_{0 x} & \cos \alpha_{0 x} \cos \alpha_{0 y}
\end{array}\right]
$$

The coordinate frame for link 1 is located at its distal end, and hence relative to link 0 it is a rotation about its joint axis followed by another rotation due to the curvature of the link. With the joint rotating $\theta_{1}$ degrees about the $z$-axis, followed by the link bending $\alpha_{1}$ degrees about the $x$-axis, the frame of link 1 relative to link 0 is represented by equation (3) where $i=1$. Rotations for links 2 and 3 are represented likewise with $i=2$ and $i=3$, respectively.

$$
{ }^{i-1} R_{i}=\left[\begin{array}{ccc}
\cos \theta_{i} & -\sin \theta_{i} \cos \alpha_{i} & \sin \theta_{i} \sin \alpha_{i} \\
\sin \theta_{i} & \cos \theta_{i} \cos \alpha_{i} & -\cos \theta_{i} \sin \alpha_{i} \\
0 & \sin \alpha_{i} & \cos \alpha_{i}
\end{array}\right]
$$

\subsection{Design parameters}

As the human maneuvers their upper limb the exoskeleton is required to position link 3 in the appropriate location relative to the humerus. This is achieved during operation by controlling the joint angles $\theta_{1}, \theta_{2}$ and $\theta_{3}$. However from the previous equations it is obvious that parameters $\alpha_{0 x}, \alpha_{0 y}, \alpha_{1}, \alpha_{2}$ and $\alpha_{3}$ also play an important role. These are the design parameters which will be optimised to maximise the exoskeleton ROM. Upper and lower bounds for these parameters can be set using appropriate rationale based on the exoskeleton's design. Parameters $\alpha_{0 x}$ and $\alpha_{0 y}$ determine the location of joint 1 relative to the torso. We limit $\alpha_{0 x}$ to between $-45^{\circ}$ and $90^{\circ}$ as outside these bounds joint 1 is likely to interfere with the torso or the arm during anterior and posterior reaching motions, as shown in Figure 4a. Similarly we limit $\alpha_{0 y}$ to between $0^{\circ}$ and $45^{\circ}$, as outside these bounds joint 1 is likely to interfere with the neck or the arm during lateral reaching motions as shown in Figure 4b.

Parameters $\alpha_{1}$ and $\alpha_{2}$ define the bend arc angle in links 1 and 2. As these angles approach $0^{\circ}$ or $180^{\circ}$ the joint axes become aligned and a kinematic singularity occurs, as shown in Figure 4c. We limit these values to be between $20^{\circ}$ and $160^{\circ}$. 


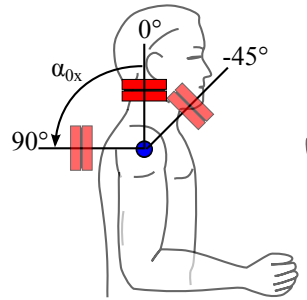

(a)

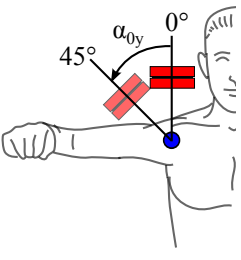

(b)

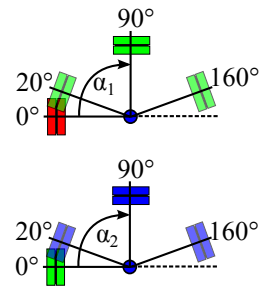

(c)

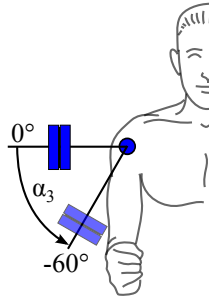

(d)

Fig. 4 Exoskeleton design parameters. (a) $\alpha_{0 x}$ defines the anterior/posterior location of joint 1 . (b) $\alpha_{0 y}$ defines the lateral/medial location of joint 1. (c) $\alpha_{1}$ and $\alpha_{2}$ define the arc angle of links 1 and 2. (d) $\alpha_{3}$ defines the angle between joint 3 and the humerus.

We constrain joint 3 to be located medially to the humerus because if positioned anteriorly it will collide with the torso during medial shoulder rotation. If located posteriorly it will collide during lateral rotation, hence locating it laterally to the humerus is the most practical. With joint 3 confined to this plane it is possible to define an angle between it and the humerus, as is done by examples in the literature $[9,3]$. Parameter $\alpha_{3}$ defines the angle that joint 3 makes with the humerus which we allow to be in the range $-60^{\circ}$ to $0^{\circ}$ as shown in Figure $4 \mathrm{~d}$. All of the design parameters are arranged into set $D P=\left\{\alpha_{0 x}, \alpha_{0 y}, \alpha_{1}, \alpha_{2}, \alpha_{3}\right\}$ which are summarised in Table 2 along with their upper and lower bounds.

Table 2 Summary of exoskeleton design parameters and their bounds used for optimisation.

\begin{tabular}{clrr}
\hline Symbol & Description & Lower bound & Upper bound \\
\hline$\alpha_{0 x}$ & Link 0 $x$-axis rotation & $-45^{\circ}$ & $90^{\circ}$ \\
$\alpha_{0 y}$ & Link 0 y-axis rotation & $0^{\circ}$ & $45^{\circ}$ \\
$\alpha_{1}$ & Link 1 bend arc angle & $20^{\circ}$ & $160^{\circ}$ \\
$\alpha_{2}$ & Link 2 bend arc angle & $20^{\circ}$ & $160^{\circ}$ \\
$\alpha_{3}$ & Link 3 bend arc angle & $-60^{\circ}$ & $0^{\circ}$ \\
\hline
\end{tabular}

\subsection{Exoskeleton Range of Motion (ROM)}

The human humerus cannot achieve every possible orientation in 3D space as it is subject to its own physiological constraints, hence the exoskeleton is only required to reach the orientations within the ROM of the human. The ROM of the exoskeleton is quantified by the percentage of the human ROM (i.e. $\psi_{H}$ ) which it can successfully reach. Three criteria determine whether or not the exoskeleton can reach a humerus orientation for a given set of design parameters. Firstly we check 
if an inverse kinematic solution exists. If a solution does exist, we check if this solution causes any collision between the exoskeleton and the wearer. Lastly we also evaluate if the robot is considered too close to the singularity condition.

\subsubsection{Inverse kinematic solution}

For each humerus orientation in set $\psi_{H}$ we solve for the exoskeleton joint angles $\theta_{1}, \theta_{2}, \theta_{3}$. Because the position of joints 1 and 3 are defined by the exoskeleton design parameters and the humerus orientation, we can check if an inverse kinematic solution exists by whether of not it is able to reach the angular distance required between these two joints. The $z$-axis of ${ }^{G} R_{0}$ is the axis of joint 1 which we define as $\mathbf{z}_{0}$. Similarly the $z$-axis of ${ }^{G} R_{2}$ is the axis of joint 3 which we define as $\mathbf{z}_{2}$. The maximum angle the exoskeleton can produce between its first and third joint axes is $\alpha_{1}+\alpha_{2}$ when $\theta_{2}=0$. The minimum angle is $\left|\alpha_{1}-\alpha_{2}\right|$ when $\theta_{2}=\pi$. From the angle required (i.e. $\cos ^{-1}\left(\mathbf{z}_{0} \cdot \mathbf{z}_{2}\right)$ ) we use (4) and (5) to determine if the exoskeleton can reach a specific humerus orientation for a given set of exoskeleton design parameters.

$$
\begin{gathered}
\mathbf{z}_{0}=\left[\begin{array}{c}
\sin \alpha_{0 y} \\
-\sin \alpha_{0 x} \cos \alpha_{0 y} \\
\cos \alpha_{0 x} \cos \alpha_{0 y}
\end{array}\right] \quad \mathbf{z}_{2}=R_{H}\left[\begin{array}{c}
\cos \alpha_{3} \\
0 \\
\sin \alpha_{3}
\end{array}\right] \\
c_{1}\left(R_{H}, D P\right)= \begin{cases}0, & \text { if }\left|\alpha_{1}-\alpha_{2}\right|<\cos ^{-1}\left(\mathbf{z}_{0} \cdot \mathbf{z}_{2}\right)<\alpha_{1}+\alpha_{2} \\
1, & \text { otherwise }\end{cases}
\end{gathered}
$$

If a solution does exist, it can be solved using inverse kinematics methods. However we need to consider that when a solution does exist there are typically two solutions, i.e $\pm \theta_{2}$. To handle this we define the exoskeleton as operating in either one of two modes. Mode 1 has $\theta_{2}$ in the range $0<\theta_{2}<\pi$, and mode 2 the range $-\pi<\theta_{2}<0$. As the robot transitions between these modes $\left(\theta_{2}=0\right.$ or $\left.\theta_{2}=\pi\right)$ the exoskeleton becomes singular. During operation the robot should operate solely in one mode. By specifying which mode the exoskeleton operates in allows kinematic solutions that are consistent during operation to be analysed during optimisation.

\subsubsection{Collision}

Every inverse kinematic solution is checked for collisions between the exoskeleton and the human. If a collision is present, then we identify that the exoskeleton is unable to reach the corresponding humerus orientation $R_{H}$ for the given design parameters $D P$. This is formalised by equation (6).

$$
c_{2}\left(R_{H}, D P\right)= \begin{cases}0, & \text { if no collision present } \\ 1, & \text { if collision present }\end{cases}
$$


To implement this requires a fast and efficient method for performing collision checking between the exoskeleton and the wearer during the optimisation. Methods that represent both the exoskeleton and human as solid meshes and then perform mesh-mesh collision checking were found to be too computationally expensive. Instead a simpler method was used based on a sphere with its centre at the shoulder, as seen in Figure 5. On this sphere two regions were defined where if the exoskeleton was located it is likely to be colliding with the human. The first region represented collision between the exoskeleton and the upper arm. A point was created where the line connecting the shoulder and elbow intersect the sphere, hence this point varied for different humerus orientations. Any points on the sphere what are within a defined distance to this point are said to be within the region and hence colliding with the humerus. The second region represented collision between the exoskeleton and the torso. This region was defined using two points on the sphere which remained static regardless of humerus orientation. Any point on the sphere within a defined distance to either of these two points, or to the shortest arc joining them, were said to be within this region and hence colliding with the torso. The joint axes of the exoskeleton are projected onto the sphere surface, and if they are located within either of these regions then the exoskeleton is deemed to be colliding. To account for collisions of the links, mid points between each joint axis were also checked. This method was efficient to calculate and hence feasible for use in the optimisation.

Fig. 5 Robot-human collision model defines regions on a sphere about the shoulder in which if the exoskeleton joints or links were located, they are likely to collide with the torso or upper arm.
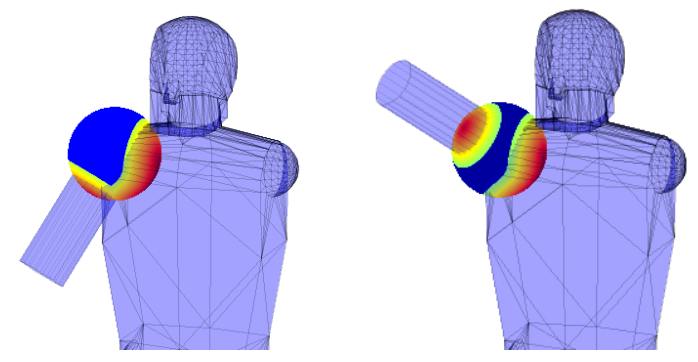

\subsubsection{Singularity}

The exoskeleton enters a kinematic singularity when all the joint axes lay in a plane, reducing the workspace degrees of freedom from three to two. For each inverse kinematic solution the singularity condition is checked. Several singularity measures based on the manipulability of the robot have been developed, many related to the Jacobian [15]. The $3 \times 3$ Jacobian matrix $\mathbf{J}$ relating joint velocity to angular workspace velocity is calculated from the axis of rotation of each joint using (7).

$$
\mathbf{J}=\left[{ }^{G} R_{0}\left[\begin{array}{l}
0 \\
0 \\
1
\end{array}\right]\left|{ }^{G} R_{0}{ }^{0} R_{1}\left[\begin{array}{l}
0 \\
0 \\
1
\end{array}\right]\right|{ }^{G} R_{0}{ }^{0} R_{1}{ }^{1} R_{2}\left[\begin{array}{l}
0 \\
0 \\
1
\end{array}\right]\right]
$$


We define the eigenvalues of $\mathbf{J}$ as $\lambda_{1}, \lambda_{2}$ and $\lambda_{3}$. The square root of the minimum eigenvalue corresponds to the minimum workspace velocity that can be achieved by a unit joint velocity vector, which at singular configurations reduces to zero [15]. The singularity of each inverse kinematic solution is analysed by comparing the minimum eigenvalue square root with a threshold value $\varepsilon$. If it is less than this threshold then it is considered as being too close to singularity and hence $R_{H}$ cannot be reached for the given set of design parameters $D P$, formalised by equation (8).

$$
c_{3}\left(R_{H}, D P\right)= \begin{cases}0, & \text { if } \min \left(\sqrt{\lambda_{1}}, \sqrt{\lambda_{2}}, \sqrt{\lambda_{3}}\right)>\varepsilon \\ 1, & \text { if } \min \left(\sqrt{\lambda_{1}}, \sqrt{\lambda_{2}}, \sqrt{\lambda_{3}}\right) \leq \varepsilon\end{cases}
$$

\section{Optimisation Model and Results}

To find the optimal design we create a fitness function to calculate how much of the human ROM the exoskeleton design can reach. Each element in the humerus orientation set $\psi_{H}$ is tested for validity based on inverse kinematic solution (5), collision (6), and singularity (8). Using (9) a subset $\psi_{E}$ is created containing the valid orientations the exoskeleton can reach with all three conditions satisfied. The ROM of the exoskeleton with respect to the humerus is calculated by the number of elements in $\psi_{E}$ relative to $\psi_{H}$ (10). A fitness function (11) is defined which approaches zero as the exoskeleton ROM approaches $100 \%$. Optimisation of the fitness function was implemented in MATLAB using a genetic algorithm (GA) in the Global Optimization Toolbox. A population size of 500 was used with the inputs being the bounded design parameters detailed in Section 3.1.

$$
\begin{gathered}
\psi_{E}=\left\{\psi_{H} \mid c_{1}\left(\psi_{H}, D P\right)=0, c_{2}\left(\psi_{H}, D P\right)=0, c_{3}\left(\psi_{H}, D P\right)=0\right\} \\
\mathrm{ROM}=100 \% \times \frac{\left|\psi_{E}\right|}{\left|\psi_{H}\right|}
\end{gathered}
$$

$$
\text { Minimise } f(D P) \text {, where } f(D P)=\left|\psi_{H}\right|-\left|\psi_{E}\right|
$$

\subsection{ROM optimisation results}

Optimisation was performed with the singularity threshold $\varepsilon$ set at $0,0.1,0.2$ and 0.3 . This was repeated with the inverse kinematic solution performed in both mode 1 and mode 2, as described in Section 3.2.1. Figure 6 shows the best ROM result calculated in the population versus generation during optimisation. The corresponding optimal design parameters are shown in Table 3. It is seen that the optimised ROM result decreases as $\varepsilon$ is increased, which is expected since a larger $\varepsilon$ puts more strin- 
gent constraints on the singularity condition. Comparison of the results indicate that mode 2 is the preferred kinematic solution, obtaining a greater ROM compared to mode 1 across all $\varepsilon$ values tested.

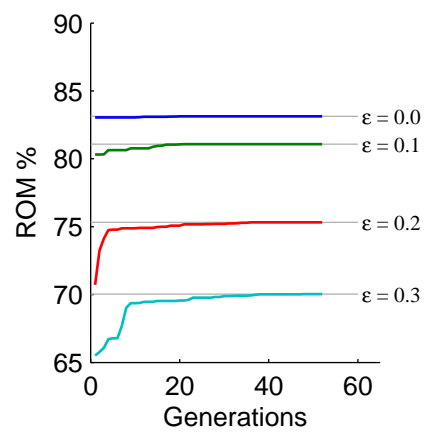

(a) Mode 1

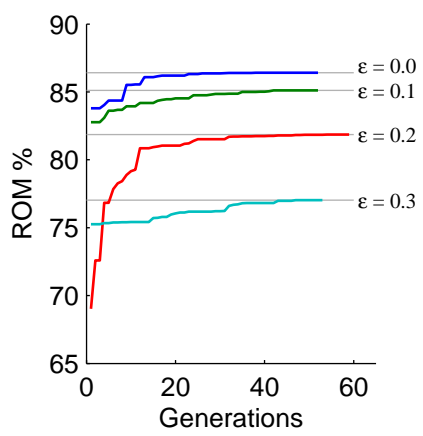

(b) Mode 2

Fig. 6 Optimisation results of the best ROM calculated by the GA versus generation. (a) Inverse kinematic solution mode 1 . (b) Inverse kinematic solution mode 2.

Table 3 Optimised design parameters and corresponding calculated ROM for both inverse kinematic solution modes and the four singularity threshold $(\varepsilon)$ values analysed.

\begin{tabular}{cccccccc}
\hline Kinematic solution & $\varepsilon$ & $\alpha_{0 x}$ & $\alpha_{0 y}$ & $\alpha_{1}$ & $\alpha_{2}$ & $\alpha_{3}$ & ROM \\
\hline Mode 1 & 0.0 & $32.6^{\circ}$ & $26.9^{\circ}$ & $45.8^{\circ}$ & $39.8^{\circ}$ & $0.0^{\circ}$ & $83.13 \%$ \\
Mode 1 & 0.1 & $27.2^{\circ}$ & $21.8^{\circ}$ & $47.3^{\circ}$ & $39.8^{\circ}$ & $0.0^{\circ}$ & $81.08 \%$ \\
Mode 1 & 0.2 & $10.2^{\circ}$ & $13.2^{\circ}$ & $48.8^{\circ}$ & $49.2^{\circ}$ & $0.0^{\circ}$ & $75.32 \%$ \\
Mode 1 & 0.3 & $8.8^{\circ}$ & $19.4^{\circ}$ & $55.0^{\circ}$ & $69.3^{\circ}$ & $-31.1^{\circ}$ & $70.04 \%$ \\
\hline Mode 2 & 0.0 & $71.5^{\circ}$ & $32.3^{\circ}$ & $63.8^{\circ}$ & $71.1^{\circ}$ & $-25.7^{\circ}$ & $86.42 \%$ \\
Mode 2 & 0.1 & $68.8^{\circ}$ & $32.2^{\circ}$ & $64.1^{\circ}$ & $72.0^{\circ}$ & $-23.3^{\circ}$ & $85.12 \%$ \\
Mode 2 & 0.2 & $70.5^{\circ}$ & $33.6^{\circ}$ & $65.2^{\circ}$ & $75.1^{\circ}$ & $-28.3^{\circ}$ & $81.86 \%$ \\
Mode 2 & 0.3 & $67.7^{\circ}$ & $35.7^{\circ}$ & $67.5^{\circ}$ & $80.0^{\circ}$ & $-38.7^{\circ}$ & $77.02 \%$ \\
\hline
\end{tabular}

As a comparison an exhaustive method was implemented using a $10^{\circ}$ step size to search through design parameter combinations. This was done for kinematic mode 2 with $\varepsilon=0$. This approach took around 15.5 hours to complete using an 8 core high performance computer, much longer than the GA which took approximately 175 minutes using the same computer setup. The best result using the exhaustive search was a ROM of $83.7 \%$, less than the $86.42 \%$ result using the GA. This increases our confidence that the GA found a solution close to the global optimum. 


\subsection{Task-specific optimisation}

In specific applications having an exoskeleton reach the entire human ROM may not be necessary. For tasks that only require a small part of this workspace, it could be beneficial to maximise the exoskeleton's ROM in this smaller subspace relevant to the task. This may allow a large percentage of the ROM subset to be reached, while allowing the optimisation to achieve a design with better secondary characteristics, for example more stringent singularity constraints. We demonstrate this by dividing the frontal workspace into two areas as shown in Figure 7. We can imagine that these two areas corresponds to two different tasks. Task $\mathrm{A}$ has the humerus between $0^{\circ}$ and $70^{\circ}$ in the anterior-lateral workspace as shown in Figure 7a. Task B has the humerus between $70^{\circ}$ and $180^{\circ}$ in the anterior-medial workspace as shown in Figure $7 \mathrm{~b}$. Subsets of the human ROM are then created by extracting humerus orientations that lay within these two areas. We apply the optimisation to maximise the exoskeleton ROM towards these two humerus ROM subsets.

Results for the task-specific optimisation, calculated with $\varepsilon=0.3$ and kinematic mode 2, are shown in Table 4. The ROM achieved was $93.57 \%$ and $99.67 \%$ for the tasks A and B respectively. Even with a stringent singularity threshold of $\varepsilon=0.3$ the ROM obtained for these two specific tasks is much larger than the ROM obtained when attempting to achieve the equivalent to a complete human ROM, which was $86.42 \%$ with the most relaxed singularity constraint, i.e. $\varepsilon=0$.

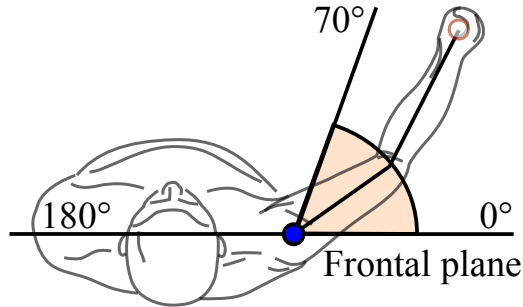

(a) Task A

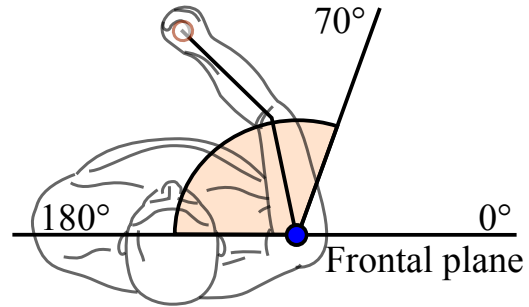

(b) Task B

Fig. 7 Two subsets of the humerus ROM are created by dividing the frontal workspace into regions corresponding to different conceptual tasks.

Table 4 Optimised design parameters and corresponding calculated ROM when optimising the exoskeleton design for a subset of human ROM corresponding to two different tasks. Results were calculated for inverse kinematic solution mode 2 and singularity threshold $\varepsilon=0.3$.

\begin{tabular}{ccccccc}
\hline Task & $\alpha_{0 x}$ & $\alpha_{0 y}$ & $\alpha_{1}$ & $\alpha_{2}$ & $\alpha_{3}$ & ROM \\
\hline A & $90.0^{\circ}$ & $31.2^{\circ}$ & $59.6^{\circ}$ & $63.4^{\circ}$ & $-39.8^{\circ}$ & $93.57 \%$ \\
B & $75.1^{\circ}$ & $45.0^{\circ}$ & $67.3^{\circ}$ & $82.4^{\circ}$ & $-27.7^{\circ}$ & $99.67 \%$ \\
\hline
\end{tabular}




\section{Conclusion}

The optimisation of the exoskeleton design was shown to maximise its ROM towards that achievable by the natural human humerus. The method used allowed factors such as collisions between exoskeleton and human, as well as an adjustable threshold on kinematic singularity to be considered during the optimisation. Unlike the commonly used approach of manually optimising a shoulder exoskeleton design by positioning the singularity, the optimisation method automatically determined the optimal singularity location, as well as additional design parameters such as the arc angle of the links. It was also demonstrated how the exoskeleton design can be optimised considering a subset of human ROM relevant to specific tasks. This highlights how the presented optimisation method can be a useful tool for the creation or modification of new and existing exoskeleton designs for use in specialised industrial or service applications.

For the optimisation to be made feasible several simplifications were made, for example the assumption of the shoulder behaving as a pure spherical joint. Using a similar optimisation framework as the one presented, more sophisticated methods can be incorporated. Future work will look at extending the optimisation using more advanced methods such as mesh-mesh approaches for realistic collision checking, as well as applying the optimisation on alternative exoskeleton designs. Our goal is to develop an upper limb exoskeleton platform with a large ROM for researching new assistive paradigms $[5,4]$.

\section{Appendix - Biomechanical Model Inverse Kinematics}

For a given humerus orientation defined by rotation matrix $R_{H}$ we solve for the corresponding joint angles $\phi_{A}, \phi_{F}$ and $\phi_{R}$ for the biomechanical shoulder model [12]. There actually exists two solutions for these angles that provide the specified humerus orientation.

$$
\begin{aligned}
& \text { Solution 1: } \\
& \phi_{A}=\operatorname{atan} 2\left(-R_{H 13}, R_{H 33}\right) \\
& \phi_{F}=\operatorname{asin}\left(-R_{H 23}\right) \\
& \phi_{R}=\operatorname{atan} 2\left(-R_{H 21}, R_{H 22}\right)
\end{aligned}
$$

$$
\begin{aligned}
& \text { Solution 2: } \\
& \phi_{A}=\operatorname{atan} 2\left(R_{H 13},-R_{H 33}\right) \\
& \phi_{F}=\pi-\operatorname{asin}\left(-R_{H 23}\right) \\
& \phi_{R}=\operatorname{atan} 2\left(R_{H 21},-R_{H 22}\right)
\end{aligned}
$$

To determine if $R_{H}$ is a feasible orientation, we solve for both solutions. Then, if either solution is found to satisfy equation (1) then we consider $R_{H}$ as a feasible humerus orientation. $R_{H i j}$ is the element in row $i$ and column $j$. 


\section{References}

1. Ball, S., Brown, I., Scott, S.: Medarm: a rehabilitation robot with 5dof at the shoulder complex. In: Advanced intelligent mechatronics, 2007 IEEE/ASME international conference on, pp. 16 (2007). DOI 10.1109/AIM.2007.4412446

2. Carignan, C., Liszka, M., Roderick, S.: Design of an arm exoskeleton with scapula motion for shoulder rehabilitation. In: Advanced Robotics, 2005. ICAR '05. Proceedings., 12th International Conference on, pp. 524-531 (2005)

3. Carignan, C., Tang, J., Roderick, S.: Development of an exoskeleton haptic interface for virtual task training. In: Intelligent Robots and Systems, 2009. IROS 2009. IEEE/RSJ International Conference on, pp. 3697-3702 (2009)

4. Carmichael, M., Liu, D.: Estimating physical assistance need using a musculoskeletal model. Biomedical Engineering, IEEE Transactions on 60(7), 1912-1919 (2013)

5. Carmichael, M.G., Liu, D.: Towards using musculoskeletal models for intelligent control of physically assistive robots. In: Engineering in Medicine and Biology Society,EMBC, 2011 Annual International Conference of the IEEE, pp. 8162 -8165 (2011)

6. Carmichael, M.G., Liu, D., Waldron, K.J.: Investigation of reducing fatigue and musculoskeletal disorder with passive actuators. In: Intelligent Robots and Systems (IROS), 2010 IEEE/RSJ International Conference on, pp. $2481-2486$ (2010)

7. Engin, A.E.: On the biomechanics of the shoulder complex. Journal of Biomechanics 13(7), 575-90 (1980)

8. Hanlon, M.: Raytheon XOS 2: second generation exoskeleton. www.gizmag.com/raytheonsignificantly-progresses-exoskeleton-design/16479/ (2010). [Online; viewed 01.09.2013]

9. Johnson, G.R., Carus, D.A., Parrini, G., Scattareggia Marchese, S., Valeggi, R.: The design of a five-degree-of-freedom powered orthosis for the upper limb. Proc Inst Mech Eng H 215(3), $275-284(2001)$

10. Klein, J., Spencer, S., Allington, J., Bobrow, J., Reinkensmeyer, D.: Optimization of a parallel shoulder mechanism to achieve a high-force, low-mass, robotic-arm exoskeleton. Robotics, IEEE Transactions on 26(4), 710-715 (2010)

11. Klopčar, N., Tomšič, M., Lenarčič, J.: A kinematic model of the shoulder complex to evaluate the arm-reachable workspace. J Biomech 40(1), 86-91 (2007)

12. Lenarčič, J., Umek, A.: Simple model of human arm reachable workspace. Systems, Man and Cybernetics, IEEE Transactions on 24(8), 1239 -1246 (1994)

13. Lo, H.S., Xie, S.Q.: Exoskeleton robots for upper-limb rehabilitation: state of the art and future prospects. Med Eng Phys 34(3), 261 - 268 (2012)

14. Mihelj, M., Nef, T., Riener, R.: Armin ii - 7 dof rehabilitation robot: mechanics and kinematics. In: Robotics and Automation, 2007 IEEE International Conference on, pp. 4120-4125 (2007)

15. Murray, R.M., Li, Z., Sastry, S.S.: A Mathematical Introduction to Robotic Manipulation, 1 edn. CRC Press (1994)

16. Perry, J., Rosen, J., Burns, S.: Upper-limb powered exoskeleton design. Mechatronics, IEEE/ASME Transactions on 12(4), 408-417 (2007)

17. Raytheon: Time magazine names the XOS 2 exoskeleton most awesomest invention of 2010. http://www.raytheon.com/newsroom/technology/rtn08_exoskeleton/ (2010). [Online]

18. Toyama, S., Yamamoto, G.: Development of wearable-agri-robot - mechanism for agricultural work. In: Intelligent Robots and Systems, 2009. IROS 2009. IEEE/RSJ International Conference on, pp. $5801-5806$ (2009)

19. Yamamoto, K., Ishii, M., Noborisaka, H., Hyodo, K.: Stand alone wearable power assisting suit - sensing and control systems. In: Robot and Human Interactive Communication, 2004. ROMAN 2004. 13th IEEE International Workshop on, pp. 661 - 666 (2004) 